\title{
Low frequency ac conduction and dielectric relaxation in poly(N-methyl pyrrole)
}

\author{
AMARJEET K NARULA , RAMADHAR SINGH* and SUBHAS CHANDRA \\ National Physical Laboratory, Dr K S Krishnan Marg, New Delhi 110 012, India \\ ${ }^{\ddagger}$ Department of Physics, Maitreyi College (Delhi University), Chanakyapuri, New Delhi 110 021, India
}

\begin{abstract}
The ac conductivity and dielectric constant of poly(N-methyl pyrrole) thin films have been investigated in the temperature range $77-350 \mathrm{~K}$ and in the frequency range $10^{2}-10^{6} \mathrm{~Hz}$. The well defined loss peaks have been observed in the temperature region where measured ac conductivity approaches dc conductivity. These loss peaks are associated with the hopping of the charge carriers. The frequency and temperature dependence of ac conductivity have been qualitatively explained by considering the contribution from two mechanisms; one giving a linear dependence of conductivity on frequency and other having distribution of relaxation times giving rise to broad dielectric loss peak.
\end{abstract}

Keywords. Poly(N-methyl pyrrole); dielectric relaxation; ac conduction.

\section{Introduction}

The emergence of conducting polymers as a new class of materials that can be doped to vary from semiconducting to metallic regime has offered new concepts of mechanism of charge transport. The common feature of almost all the electroactive polymers such as polypyrrole, polythiophene, polyaniline, poly( $p$-phenylene), etc is an extended localization in their polymer backbone. In polypyrrole, one dimensional chain of identical monomer units has $\pi$ electrons but upon polymerization the spatial extent of these electrons is influenced by significant overlap and the electrons become delocalized over the length of the polymer chain in the form of bands analogous to that of a semiconductor and the distortion of the polymer chain around the injected charge carriers leads to formation of polarons (Reghu et al 1991). Polypyrrole (PPY) family of polymers is one of the best candidate materials for various device applications such as in solar cells, electromagnetic shielding, electrodes for rechargeable batteries, sensors, etc. Hence it is worthwhile to examine the mechanism of charge transport in this family of polymers. A review of literature suggests that not much attention has been paid to lightly doped samples, perhaps the main aim of the researcher in this field is to enhance the conductivity. To have an overview of the mechanism of charge transport in poly(N-methyl pyrrole) $[\mathrm{P}(\mathrm{NMPY})]$, we report here its dielectric constant and ac conductivity behaviour as a function of frequency and temperature. The dielectric and conductivity relaxations in other conducting polymers

\footnotetext{
*Author for correspondence

${ }^{\dagger}$ Paper presented at the 5th IUMRS ICA98, October 1998, Bangalore
}

have been discussed and analyzed in light of the model of multiple conductivity relaxation modes (Ito et al 1992). On the basis of multiple relaxation modes observed in these materials a model has been proposed which suggests the application of two relaxation mechanisms to explain the temperature and frequency dependence of conductivity in $\mathrm{P}(\mathrm{NMPY})$.

\section{Experimental}

Films of poly(N-methyl pyrrole) (thickness $\sim 6-17 \mu \mathrm{m}$ ) were prepared by electrochemical polymerization technique. Films were deposited on indium-tin oxide coated glass plate by electrochemical polymerization of $0.1 \mathrm{M}$ doubly distilled $\mathrm{N}$-methyl pyrrole in the solvent propylene carbonate and electrolyte tetraethyl ammonium tetrafluoroborate. Before polymerization the reaction mixtures were thoroughly degassed by passing dry nitrogen. The deposition was carried out at $273 \mathrm{~K}$ in an inert atmosphere at the polymerization current density of $1 \mathrm{~mA} / \mathrm{cm}^{2}$ for $40-130 \mathrm{~min}$. The fully doped films were peeled off, thoroughly washed in deionized water and dedoped in ammonia for different intervals of time in order to obtain the films of different $\mathrm{BF}_{4}^{-}$ion concentrations and hence having different values of conductivity. The details of estimation of $\mathrm{BF}_{4}^{-}$ions have been given elsewhere (Singh et al 1996a, b). We are reporting the results on the film having $\mathrm{BF}_{4}^{-}$ion concentration of $17 \cdot 1 \%$ and room temperature $(300 \mathrm{~K})$ dc conductivity of $1.20 \times$ $10^{-4} \Omega^{-1} \mathrm{~cm}^{-1}$. Gold was vacuum deposited on both sides of the samples making an $\mathrm{Au}-\mathrm{P}-\mathrm{Au}$ structure. The vacuum deposition of gold yielded ohmic contact which is evident from the linear nature of $I-V$ characteristics shown in figure 1. Ac conductivity and dielectric constant were 
measured in a specially designed three-terminal cell in the temperature region $77-350 \mathrm{~K}$ by using HP 4192A LF impedance analyzer in the frequency range $100 \mathrm{~Hz}-$ $1 \mathrm{MHz}$. The dc conductivity was measured with the help of Keithley's 617 electrometer, 220 constant current source and 2000 DMM. Transmission electron micrographs and electron diffraction patterns were recorded with the help of transmission electron microscope model JEM-200 CX. Electron spin resonance (ESR) measurements were performed by Jeol JES-FE 3XG spectrometer operating in X-band mode.

\section{Results and discussions}

The dielectric parameters were evaluated by measuring equivalent parallel capacitance $C_{\mathrm{p}}$ and dissipation factor $\tan \delta(D)$ or the equivalent resistance $R_{\mathrm{p}}$ of the sample by using the equations

$$
\varepsilon^{\prime}=C_{\mathrm{p}} / C_{0}, \varepsilon^{\prime \prime}=\varepsilon^{\prime} / \omega C_{\mathrm{p}} R_{\mathrm{p}} \text { or } \varepsilon^{\prime \prime}=\varepsilon^{\prime} D
$$

and

$$
\sigma_{\mathrm{m}}(\omega)=2 \pi f \varepsilon^{\prime \prime}
$$

where

$$
C_{0}=(0 \cdot 08854 X A / t) p f,
$$

is the geometrical capacitance of vacuum of the same dimensions as the sample. $A$ and $t$ are the area and thickness of the sample, respectively and $f$ the measuring frequency. $C_{\mathrm{p}}$ is the capacitance measured in $p f, \varepsilon^{\prime}$ the real dielectric constant and $\varepsilon^{\prime \prime}$ the imaginary dielectric constant. It is evident from figure 1 that the dielectric constant at a given frequency is a slowly varying function of temperature in the low temperature region and the dependence increases with increasing temperature. This behaviour is consistent with Debye-type dielectric dispersion characterized by a relaxation time $\tau$ and hence a relaxation frequency $f_{0}$. The measuring frequency is higher in the low temperature region and lower at higher temperatures than $f_{0}$. The measured ac conductivity at four fixed frequencies has been plotted as a function of temperature in figure 2. In low temperature region the measured ac conductivity is almost independent of temperature and the strong temperature dependence occurs at higher temperatures for higher frequencies. The ac dielectric loss $\varepsilon_{\mathrm{ac}}^{\prime \prime}$ derived from the relation

$$
\omega e_{0} \varepsilon_{\mathrm{ac}}^{\prime \prime}=\sigma_{\mathrm{m}}(\omega)-\sigma_{\mathrm{dc}}=\sigma_{\mathrm{ac}},
$$

where $e_{0}$ is permittivity of free space, $\sigma_{\mathrm{m}}(\omega)$ the measured ac conductivity, $\sigma_{\mathrm{dc}}$ the dc conductivity and $\sigma_{\mathrm{ac}}$ the ac conductivity, has been shown as a function of temperature in figure 3 . The comparison of figures 1 and 3 indicates that the temperature dependence of dielectric constant

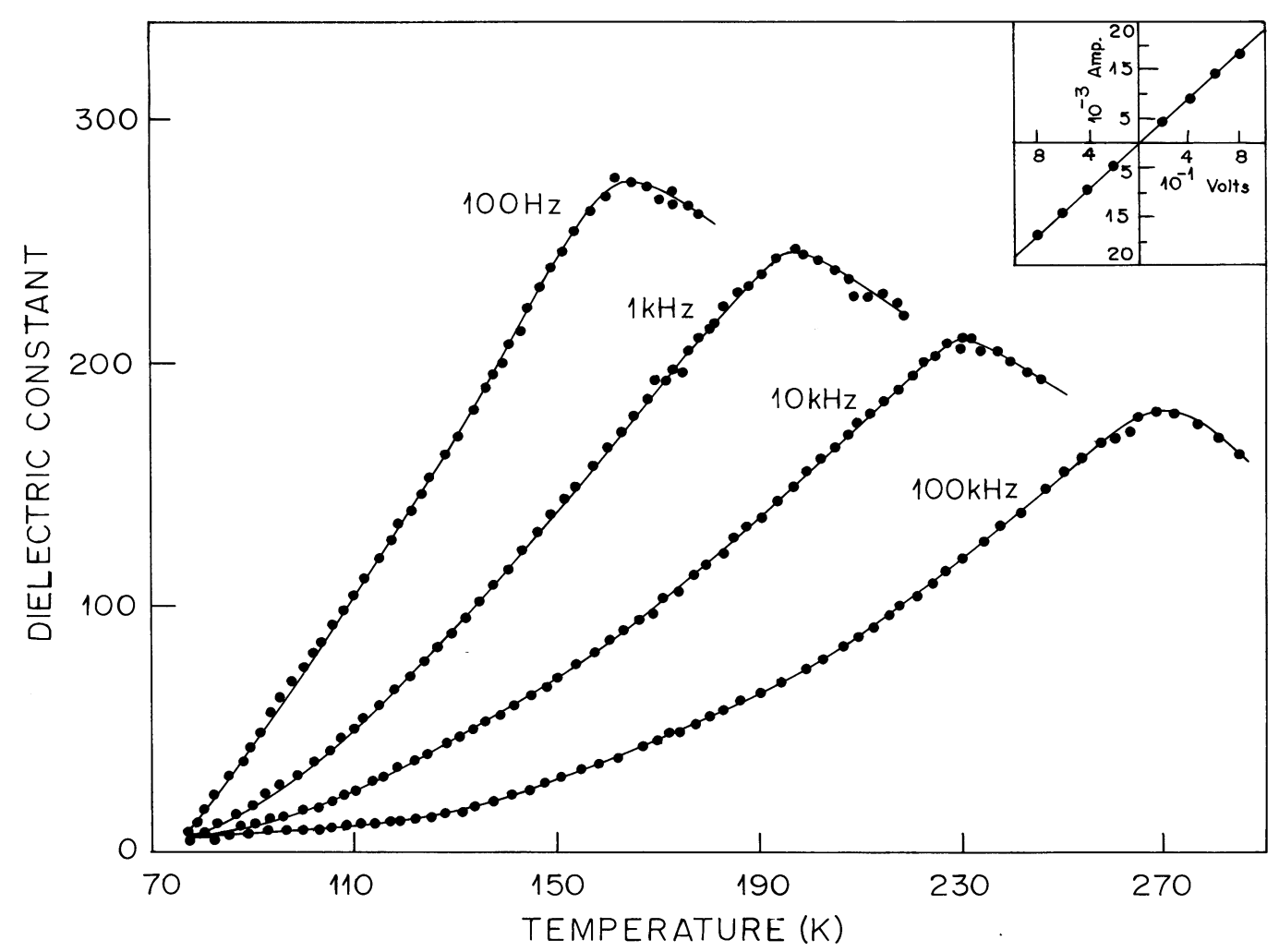

Figure 1. Dielectric constant as a function of temperature for four fixed frequencies. The inset shows $I-V$ characteristics at $300 \mathrm{~K}$. 
increases rapidly with temperature particularly in temperature region where dielectric loss peak occurs. The shift of dielectric loss peak towards higher frequency side with increasing temperature is consistent with Debye-type dispersion (figure 3). The dielectric loss has been evaluated from the measured ac conductivity values by subtracting the dc conductivity. In the region where two values become close to each other the error in dielectric loss becomes large. It can be seen here that the region where there is strong temperature dependence of $\varepsilon^{\prime}$ at a given frequency is same at which $\sigma_{\mathrm{m}}(\omega)$ approaches $\sigma_{\mathrm{dc}}$ (figure 2). Thus the variation of $\varepsilon^{\prime \prime}$ with temperature indicates that the loss peaks are real. The magnitude of the loss peak decreases and temperature at which loss peak occurs increases with increasing frequency. The loss peaks may be attributed to the motion of charge carriers at different redox centres and the other defect sites along the chain length and the transit time associated with each of these varies which causes a distribution of relaxation times (Singh et al 1996a). The dielectric constant for a Debye-type process is given by

$$
\left(\varepsilon^{\prime}-\varepsilon_{\infty}\right)=\left(\varepsilon_{0}-\varepsilon_{\infty}\right) /\left(1+\omega^{2} \tau^{2}\right),
$$

where $\varepsilon_{0}$ is the static dielectric constant and $\varepsilon_{\infty}$ the infinite frequency dielectric constant and $\omega=2 \pi f$. In the low temperature region where $f \gg f_{0}, \quad \varepsilon^{\prime} \rightarrow \varepsilon_{\infty}$ and at higher temperatures where $f \ll f_{0}, \varepsilon^{\prime} \rightarrow \varepsilon_{0}$. The relaxation

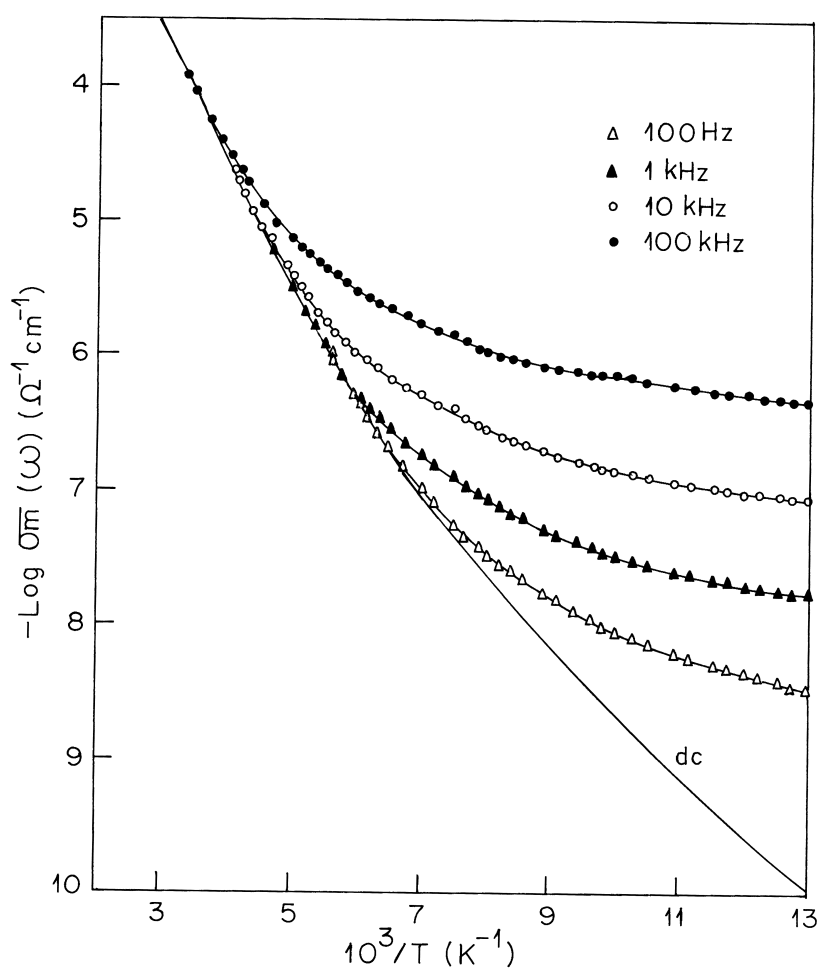

Figure 2. Dc and measured ac conductivities as functions of reciprocal temperature. frequency and static dielectric constant estimated from figure 1 have been shown as a function of temperature in figure 4 . It is evident from figure 4 that $\varepsilon_{0}$ decreases with increase in temperature. The variation of measured ac conductivity with frequency at different fixed temperatures is shown in figure 5a. It is evident from this figure that the ac conductivity at low temperatures (77$133 \mathrm{~K}$ ) is frequency dependent and can be expressed as (Mott and Davis 1979)

$$
\sigma(\omega)=A \omega^{s}
$$

where $s(0.5<s<1)$ is independent of frequency and at $77 \mathrm{~K}$ the value of $s$ is 0.73 . The density of states near the

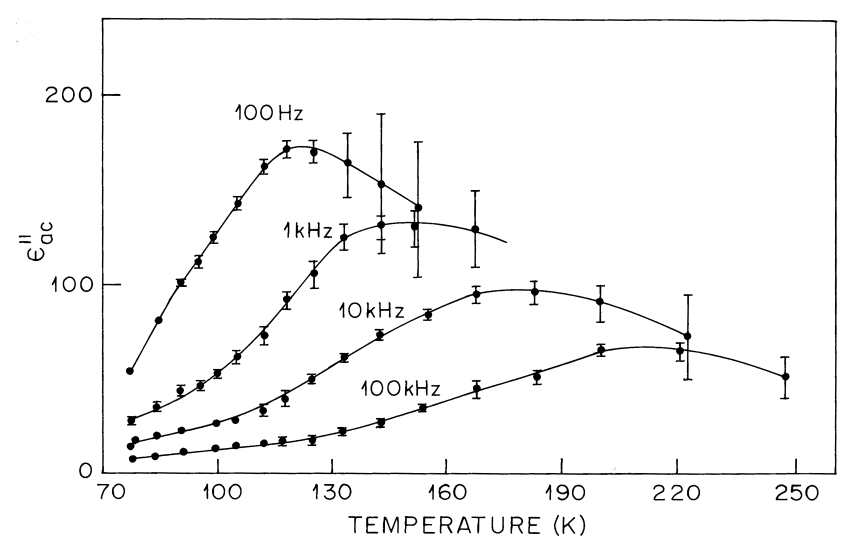

Figure 3. Dielectric loss as a function of temperature at four fixed frequencies. The vertical bars indicate uncertainty within $\pm 2 \%$.

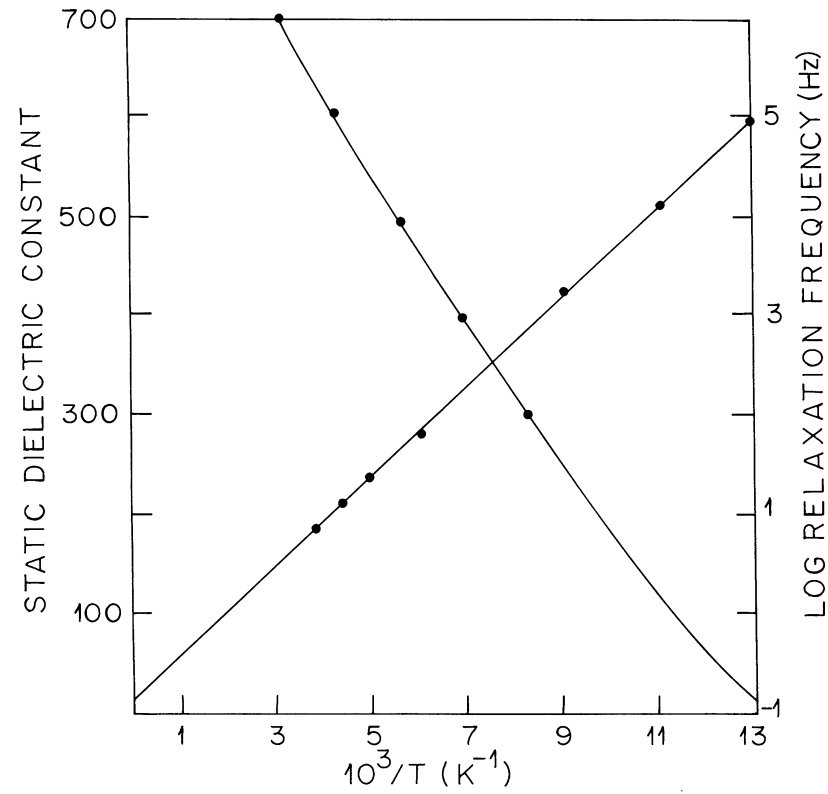

Figure 4. Static dielectric constant and relaxation frequency as functions of reciprocal temperature. 
Fermi level can be estimated from ac conductivity at low temperatures by the expression given by Austin and Mott (1969),

$$
\sigma(\omega)=(1 / 3)\left\{\pi e^{2} k_{\mathrm{B}} T\left[N\left(E_{\mathrm{F}}\right)\right]^{2} \alpha^{-5} \omega\left[\ln \left(v_{0} / \omega\right)\right]^{4}\right\},
$$

where $k_{\mathrm{B}}$ is the Boltzmann's constant, $e$ the electronic charge, $v_{0}$ a frequency factor, $\alpha^{-1}=r_{\mathrm{p}}$ is the polaron radius which is estimated to be $3.82 \AA$ for $\mathrm{P}(\mathrm{NMPY})$ (Singh et al 1996c). Assuming $v_{0}=10^{13} \mathrm{~Hz}$ and $\sigma(\omega)$ in the frequency range $100 \mathrm{~Hz}-100 \mathrm{kHz}$, the values of density of states near the Fermi level $N\left(E_{\mathrm{F}}\right)$ comes out to be $\sim 10^{18} \mathrm{~cm}^{-3} \mathrm{eV}^{-1}$. The reasonable values of $N\left(E_{\mathrm{F}}\right)$ suggests that the hopping between the pair of sites dominates the mechanism of charge transport in low temperature region (Mott and Davis 1979). The average site spacing $d$ is calculated by using Shimakawa's approach (Singh and Narula 1997) according to which the dielectric strength

$$
\varepsilon_{0}-\varepsilon_{\infty}=N e^{2} d^{2} /\left(12 k_{\mathrm{B}} T\right)
$$

where $N$ is the spin concentration obtained from ESR investigations and is found to be $5.01 \times 10^{20} \mathrm{~cm}^{-3}$ (Singh and Narula 1997). $\varepsilon_{0}$ at $300 \mathrm{~K}$ is taken from figure 4 whereas the infinite frequency dielectric constant $\varepsilon_{\infty}$ was assumed to be temperature independent and has been taken equal to the intercept $(=14)$ in figure 4 . The value obtained for the site spacing $d$ is $22.24 \AA$ which is about 8 monomer rings whereas the size of one monomer ring is $\sim 3 \AA$ (Maddison and Tansley 1992). The variation of dielectric constant, measured ac conductivity and derived dielectric loss as a function of frequency at $143 \mathrm{~K}$ is shown in figure $5 \mathrm{~b}$. It is also evident from figure 5 that in higher temperature region $(>133 \mathrm{~K})$, the measured ac conductivity in the low frequency region is independent of frequency and then increases with increase in frequency. The loss peak occurs in the same region where strong dispersion in dielectric constant is observed. A decrease in the peak value of $\varepsilon^{\prime}$ for higher frequencies and temperatures indicates that $\varepsilon_{0}$ decreases with increase in temperature (figure 1). Therefore, the observed data of dielectric constant and ac conductivity can be divided into two regions. The low temperature region where $\sigma_{\mathrm{m}}(\omega)$ is frequency dependent and $\varepsilon^{\prime}$ is almost frequency independent $(77-133 \mathrm{~K})$ and the high temperature region where $\varepsilon^{\prime}$ is frequency dependent and $\sigma_{\mathrm{m}}(\omega)$ becomes relatively weakly dependent on frequency. A decrease in slope $s$ (figure 5) has been found with increase in temperature which is due to larger increase in conductivity with temperature at lower frequencies as compared to higher frequencies. It is evident from figure 5 that the low frequency conductivity values is almost equal to dc conductivity value while in high frequency region there is sublinear dependence of conductivity on frequency. This is, however, inconsistent with Debye-type dispersion, which suggests that a single
Debye-type relaxation is inadequate to explain the dielectric data. It can be seen from figure 2 that the temperature at which the dc conductivity becomes equal to $\sigma_{\mathrm{m}}(\omega)$ for a given frequency increases with increasing frequency. The dc conductivity shows an exponential dependence on temperature while ac conductivity shows linear frequency dependence. Hence at some temperatures, the measured ac conductivity for a given frequency will appear equal to dc conductivity because $\sigma_{\mathrm{dc}}$ may be very much larger than $\sigma(\omega)$ and so $\sigma_{\mathrm{m}}(\omega)$ will appear equal to $\sigma_{\mathrm{dc}}$ within the accuracy of measurement.

The dielectric constant and ac conductivity $\sigma_{\mathrm{m}}(\omega)$ were measured for three different thicknesses (viz. 6, 10, $17 \mu \mathrm{m}$ ) while keeping the area and the other preparation conditions same and they were found to be independent of thickness of the samples within the accuracy of measurement (Singh et al 1997). So the observed dielectric relaxation at higher temperatures cannot be due to the electrode barriers or macroscopic inhomogeneities (Tomazawa 1977; Singh et al 1997). Moreover the transmission electron micrographs and electron diffraction patterns of the samples show that the films are predominantly amorphous in nature. So the chances of the observed dielectric relaxation due to microinhomogeneities are rare. Hence the dielectric relaxation observed in the present investigation represents the true bulk behaviour of the samples (Singh et al 1997). Now we present a generalized model

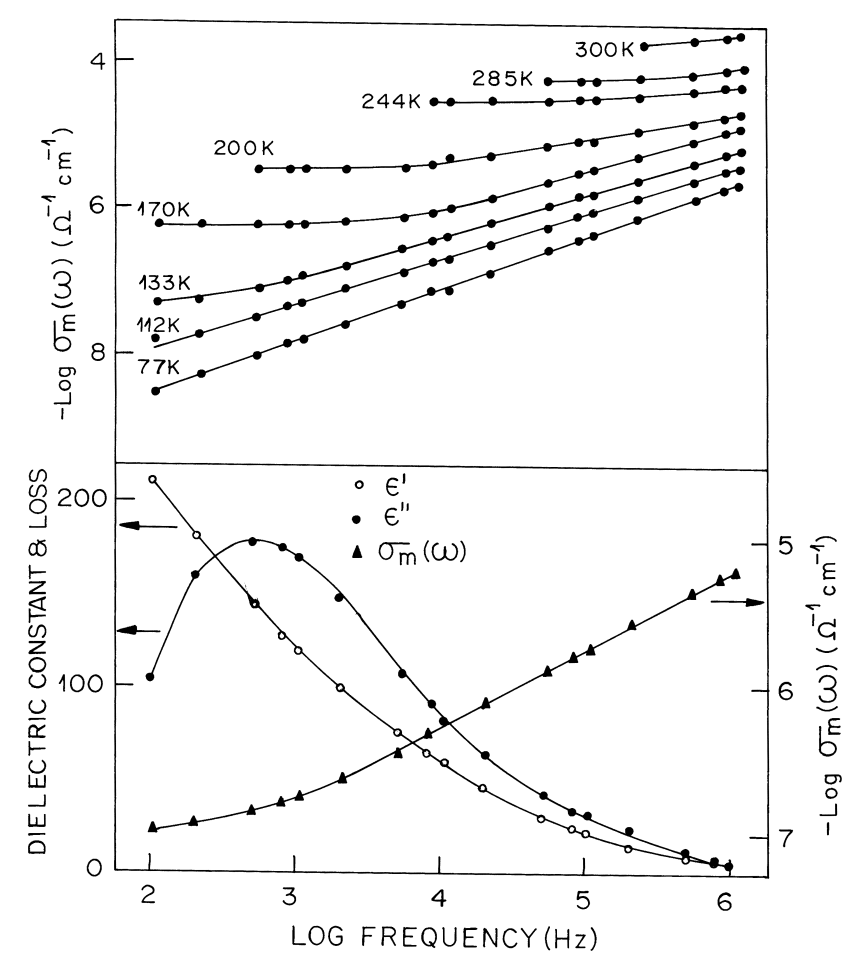

Figure 5. Measured ac conductivity as a function of frequency at different fixed temperatures (top) and dielectric constant, measured ac conductivity and dielectric loss as function of frequency at $143 \mathrm{~K}$ (bottom). 
for explaining the observed behaviour of dielectric relaxation. We have seen that at higher temperatures, for sufficiently low frequencies the ac conductivity is expected to be considerably lower than dc conductivity and the measured ac conductivity should appear frequency independent. This shows that the tail of the dielectric loss peak cannot account for the ac conductivity in the temperature region where the ac conductivity is substantially greater than dc conductivity and the experimental data cannot be explained by the models of sublinear frequency dependent conductivity. This suggests that there are two mechanisms responsible for explaining the behaviour of ac conductivity in the entire temperature range. This consists of two parts

$$
\sigma(\omega)=\sigma_{1}(\omega)+\sigma_{2}(\omega)
$$

The mechanism responsible for $\sigma_{1}(\omega)$ gives a linear dependence of conductivity on frequency of the form suggested by Austin and Mott (1969) and the mechanism responsible for $\sigma_{2}(\omega)$ gives a dielectric loss peak which is masked by dc conductivity and the dispersion is due to the distribution of relaxation times. The deviation from single Debye-type relaxation in many dielectric liquids and solids have been explained by using Cole and Cole (1941) approach according to which the complex dielectric constant $\varepsilon *$ is given by

$$
\left(\varepsilon^{*}-\varepsilon_{\infty}\right) /\left(\varepsilon_{0}-\varepsilon_{\infty}\right)=1 /\left(1+(i \omega \tau)^{\beta}\right) .
$$

The real part of the expression represents the dielectric constant and the imaginary part represents the loss or ac conductivity. The values of $\varepsilon_{0}-\varepsilon_{\infty}$ and $\tau$ (taken from figure 4) when fitted to the real part of (4) and by comparing with experimental data by using a computer program in pascal yielded the value of 0.4 for $\beta$ which was almost independent of temperature. From the imaginary part of (4) the ac conductivity is represented as

$$
\begin{aligned}
\sigma(\omega)= & \left(\omega e_{0}\left(\varepsilon_{0}-\varepsilon_{\infty}\right)(\omega \tau)^{1-\beta} \cos (\beta \pi / 2)\right) / \\
& \left(1+2(\omega \tau)^{1-\beta} \sin (\beta \pi / 2)+(\omega \tau)^{2(1-\beta)}\right) .
\end{aligned}
$$

The ac conductivity $\sigma_{1}(\omega)$ is of the form given by (2) whereas $\sigma_{2}(\omega)$ is of the form given by (5). The contribution of $\sigma_{2}(\omega)$ dominates for about 2 to 3 decades above the relaxation frequency and the contribution of $\sigma_{1}(\omega)$ dominates only at frequencies which are very much higher than the relaxation frequency where $\sigma_{\mathrm{m}}(\omega)$ is substantially higher than $\sigma_{\mathrm{dc}}$. If we assume that the slope ' $s$ ' of $\sigma(\omega)$ remains unchanged with temperature then the apparent decrease in its value is due to the contribution of $\sigma_{2}(\omega)$. Therefore, the temperature dependence of ac conductivity should then be explained by taking the contribution of both $\sigma_{1}(\omega)$ and $\sigma_{2}(\omega) . \sigma_{1}(\omega)$ at different frequencies and temperatures can be calculated by the expression

$$
\sigma_{1}(\omega)=A \omega^{s}=B T \omega^{s}
$$

where parameters $B$ and $s$ are assumed to be temperature independent. The calculated values of $\sigma_{1}(\omega)$ are shown by a dashed line in figure 6 . The experimental points are indicated by solid circles in figure 6 . It may be seen that the measured values of ac conductivity can be fully accounted for by the contribution of $\sigma_{1}(\omega)$ upto higher temperatures for higher frequencies. However, above certain temperature range the measured values become substantially greater than $\sigma_{1}(\omega)$ which is due to the fact that in this temperature region $\sigma_{2}(\omega)$ begins to dominate. The values of $\sigma_{2}(\omega)$ can be calculated by (5) for $\beta=0.4$, where $\varepsilon_{0}$ and $\tau$ at different temperatures have been taken from figure 4 . The values of $\sigma_{2}(\omega)$ have been calculated at different temperatures and frequencies and are shown by dash-dot line in figure 6 . The sum of $\sigma_{1}(\omega)$ and $\sigma_{2}(\omega)$ is shown by solid line in figure 6 . However, even a large uncertainty in $\sigma_{2}(\omega)$ at temperatures much lower than the temperature at which a peak in $\sigma_{2}(\omega)$ is observed would not be of any consequence because there the contribution of $\sigma_{1}(\omega)$ is much higher than $\sigma_{2}(\omega)$. The good agreement between theoretical and experimental values of $\sigma(\omega)$ indicates that the variation of ac conductivity can be explained satisfactorily by assuming that the two mechanisms contribute to the measured ac conductivity in which one may be associated with the hopping of the charge carriers excited to the localized band tails giving rise to dielectric loss peaks and the other to the hopping of the

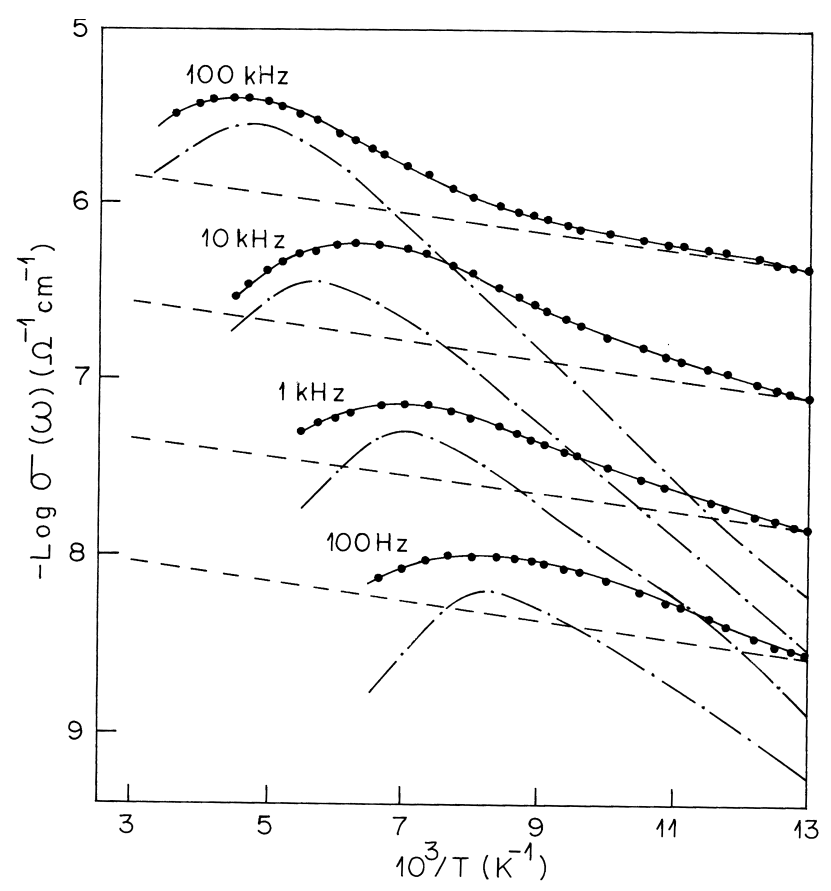

Figure 6. Dashed line shows the contribution of $\sigma_{1}(\omega)$. The dash-dot line shows the contribution of $\sigma_{2}(\omega)$. The solid line gives the contribution of $\sigma_{1}(\omega)+\sigma_{2}(\omega)$. The experimental points are marked by solid circles. 
charge carriers near the Fermi level giving a linear frequency dependent conductivity.

\section{Conclusions}

The reasonable values of density of states near Fermi level $\left(N\left(E_{\mathrm{F}}\right)\right)$ obtained from complex conductivity data suggests that the hopping between pairs of sites dominates the mechanism of charge transport in poly(N-methyl pyrrole) at low temperatures. The measured ac conductivity and dielectric constant in the entire temperature region $(77-350 \mathrm{~K})$ could arise from two distinct mechanisms based on polaronic hopping in which one gives a linear dependence of conductivity on frequency and other having a distribution of relaxation times giving rise to broad dielectric loss peaks. The average site spacing $d$ has been estimated to be $22 \cdot 24 \AA$ which suggests that the polaron localization is about 8 per pyrrole rings.

\section{Acknowledgements}

The authors are thankful to Prof A K Raychaudhuri, Director, National Physical Laboratory, New Delhi, for his keen interest. Financial Assistance from the Department of Biotechnology, New Delhi, under Grant No. BT/02/ 038/93-PID is gratefully acknowledged.

\section{References}

Austin I G and Mott N F 1969 Adv. Phys. 1841

Cole K S and Cole R H 1941 J. Chem. Phys. 9341

Ito K, Tanabe Y, Akagi K and Lancaster M 1992 Phys. Rev. B45 1246

Maddison D S and Tansley T L 1992 J. Appl. Phys. 724677

Mott N F and Davis E A 1979 Electronic processes in noncrystalline materials (London: Clarendon)

Reghu M, Subramanyam S V and Chatterjee S 1991 Phys. Rev. B43 4236

Singh R and Narula A K 1997 J. Appl. Phys. 824362

Singh R, Narula A K, Tandon R P, Mansingh A and Chandra S 1996a J. Appl. Phys. 80985

Singh R, Narula A K and Tandon R P 1996b Synth. Met. 8263

Singh R, Narula A K, Tandon R P, Mansingh A and Chandra S 1996c J. Appl. Phys. 791476

Singh R, Narula A K, Tandon R P, Mansingh A and Chandra S 1997 Philos. Mag. B75 419

Tomazawa M 1977 Treatise on material science and technology (eds) M Tomazawa and R H Doremus (New York: Academic Press) Vol. 12, p. 281 